\title{
NEW PARAMETERS OF HAND GRIP IN RHEUMATOID ARTHRITIS PATIENTS BASED ON ELECTRODYNAMIC MEASUREMENTS - A PILOT EVALUATION STUDY
}

\author{
NOVI PARAMETRI STISKA ŠAKE U BOLESNIKA S REUMATOIDNIM \\ ARTRITISOM TEMELJENI NA ELEKTRODINAMIČKIM MJERENJIMA \\ - PILOT EVALUACIJSKA STUDIJA
}

\section{Ines Doko Vajdić ${ }^{1}$, Amir Dubravić ${ }^{2}$, Ivan Michieli², Branka Medved Rogina ${ }^{2}$, Simeon Grazio ${ }^{1}$}

\author{
${ }^{1}$ University Department of Rheumatology, Physical and Rehabilitation Medicine, Sestre Milosrdnice University Hospital Center, \\ School of Medicine, Zagreb, Croatia / Klinika za reumatologiju, fizikalnu medicinu i rehabilitaciju \\ Kliničkoga bolničkog centra Sestre milosrdnice, Medicinski fakultet u Zagrebu, Zagreb, Hrvatska \\ ${ }^{2}$ Ruđer Bošković Institute, Zagreb, Croatia / Institut Ruđer Bošković, Zagreb, Hrvatska
}

Corresponding author / Adresa autora za dopisivanje:

Dr. sc. Ines Doko Vajdić, dr. med.

University Department of Rheumatology, Physical and Rehabilitation Medicine

/ Klinika za reumatologiju, fizikalnu medicinu i rehabilitaciju

Sestre Milosrdnice University Hospital Center / Klinički bolnički centar Sestre milosrdnice

Vinogradska cesta 29, 10000 Zagreb

Croatia / Hrvatska

Phone / tel.: +385-1-3787-248

Fax / faks: +385-1-3787-395

E-mail / e-pošta: inesdoko@yahoo.com

Received / Primljeno: April 30, 2020 / 30. 4. 2020. Accepted / Prihvaćeno: October 21, 2020 / 21. 10. 2020.

\section{ABstract}

Introduction: In rheumatoid arthritis, inflammation and damage lead to functional impairment. As the hand is a typical site of involvement, lower grip strength is often found in patients with rheumatoid arthritis. In these patients it is necessary to evaluate the hand grip impairment in a more detailed way. The aim of this study was to analyze a new method for measuring hand grip.

Methods: An expert electronic measuring system for obtaining dynamic time series of hand grip force was developed. We tested it in a sample of 24 participants, 12 patients with rheumatoid arthritis and 12 age- and sex-matched healthy controls. The main dynamometric parameters obtained were: maximum grip force, fatigue, grip velocity, and functional potential. These were measured at the baseline and at 60, 120, and 180 days.

Results: Compared to the control group, at baseline the patients with rheumatoid arthritis had a significantly lower maximum grip force (68.2 vs. 97.3), earlier occurrence of fatigue (22.4 vs. 24.9$)$, as well as lower grip velocity (14.8 vs. 22.2) and functional potential (808.2 vs. 1876.3). A significant improvement in all measured variables was observed during the follow-up period.

Conclusion: In our pilot study we tested a newly developed electrodynamometer measuring system and found that it can provide an objective and detailed description of the hand condition. This system has the potential to be used as a relevant indicator of hand function in patients with rheumatoid arthritis as well as to help tailor their rehabilitation.

Key words: Arthritis, rheumatoid - physiopathology, rehabilitation; Hand - physiopatholoy; Hand strength; Muscle strength dynamometer; Pilot projects

\section{SAŽETAK}

Uvod: U reumatoidnom artritisu upala i oštećenje dovode do funkcionalne nesposobnosti. Uobičajeno mjesto zahvaćenosti jest šaka i zato ovi bolesnici često imaju slabiju snagu stiska. U bolesnika s reumatoidnim artritisom potrebno je provesti detaljniju evaluaciju oštećenja šake. Cilj ove studije bila je analiza nove metode mjerenja snage stiska šake. 
Metode: Razvijen je ekspertni elektronički mjerni sustav za dobivanje dinamičkih vremenskih serija sile stiska šake. Testirali smo ga na uzorku od 24 sudionika: 12 bolesnika s reumatoidnim artritisom i 12 zdravih kontrolnih ispitanika koji se podudaraju po dobi i spolu. Glavni dobiveni dinamometrijski parametri bili su: maksimalna sila stiska šake, zamor šake, brzina stiska te funkcionalni potencijal. Navedeni parametri izmjereni su pri početnomu mjerenju i nakon 60, 120 i 180 dana.

Rezultati: U usporedbi s kontrolnom skupinom bolesnici s reumatoidnim artritisom imali su znatno manju maksimalnu silu stiska šake (68,2 prema 97,3), raniju pojavu zamora šake (22,4 prema 24,9$)$, manju brzinu stiska $(14,8$ prema 22,2$)$ i manji funkcionalni potencijal $(808,2$ prema 1876,3$)$ pri početnomu mjerenju. Tijekom praćenja primijećeno je znatno poboljšanje svih izmjerenih varijabla.

Zaključak: U našoj pilot studiji testirali smo novorazvijeni elektrodinamometrijski sustav mjerenja te smo utvrdili da se njegovom uporabom može objektivno i detaljno opisati stanje šake. Taj se sustav može upotrijebiti kao relevantan pokazatelj funkcije šake u bolesnika s reumatoidnim artritisom i može pomoći u prilagodbi njihove rehabilitacije.

KLJUČNE RIJEČI: Reumatoidni artritis - patofiziologija, rehabilitacija; Šaka - patofiziologija; Snaga šake; Dinamometar za mišićnu snagu; Pilot istraživanja

\section{INTRODUCTION}

Polyarthritis of the hand is a hallmark of rheumatoid arthritis (RA). Standard hand dynamometry registration of one-grip force or the recording of a series of multiple grips are commonly used to evaluate the function of the hand $(1,2)$. This provides an approximate measurement of handgrip strength, but not a detailed insight (3). A more detailed assessment would be useful for tailored rehabilitation of the hand in patients with RA, but also in patients with other non-rheumatic conditions involving the hand. Therefore, we developed a custom-designed electronic dynamic sensor in order to measure different minutely specified parameters for handgrip evaluation. The aim of this pilot study was to evaluate the hand grip in patients with RA using this new electrodynamic method.

\section{METHOD}

This pilot study included 24 subjects, 12 patients with RA ( 6 women and 6 men, aged 35-55 years) in the early phase of the disease, and 12 healthy sex- and age-matched controls. The patients with RA were enrolled consecutively from the outpatient clinic according to the following inclusion criteria: newly diagnosed RA with disease duration $<6$ months, age 35-55 years, and treatment with orally taken methotrexate $15 \mathrm{mg} /$ week. In the evaluation of motor hand function a new expert electronic measuring system for dynamic measurements of the hand grip force, developed at the Ruđer Bošković Institute, was used (Figure 1). The device was calibrated in accordance with hand grip comparison on a mechanical dynamometric device with the same mechanical handrail. The dynamics of the dynamometric hand grip parameters in both groups was measured at the baseline and after 60,120, and 180 days. All the patients were treated with the anchor disease-modifying anti-rheumatic drug (DMARD) methotrexate, and during the follow-up period its dosage

\section{UVOD}

Poliartritis šake jedna je od glavnih značajka reumatoidnog artritisa (RA). Za evaluaciju funkcije šake uobičajeno se upotrebljava standardna dinamometrija šake koja omogućava registraciju sile jednog stiska šake ili bilježenje serije vrijednosti višestrukih stisaka šake $(1,2)$. Tako se može dobiti okvirna vrijednost snage stiska šake, ali nedostatak ove metode jest detaljan uvid u stanje (3). Detaljnija ocjena je korisna za prilagođenu rehabilitaciju šake u bolesnika s RA, ali i za pacijente $s$ drugim nereumatskim bolestima koje zahvaćaju šake. Stoga smo razvili prilagođen elektronički, dinamički senzor da bismo mogli izmjeriti različite, detaljno određene parametre za evaluaciju stiska šake. Cilj ove pilotne studije bila je evaluacija stiska šake u bolesnika s RA uporabom nove elektrodinamičke metode.

\section{METODA}

Pilotnu studiju proveli smo na 24-ero ispitanika: 12-ero bolesnika s RA u ranoj fazi bolesti ( 6 žena i 6 muškaraca u dobi od 35 do 55 godina) i 12-ero zdravih kontrolnih ispitanika koji su se podudarali po dobi i spolu. Bolesnici s RA uključeni su u studiju uzastopno iz ambulante u skladu sa sljedećim uključnim kriterijima: nedavno postavljena dijagnoza RA s trajanjem bolesti kraćim od 6 mjeseci, dob od 35 do 55 godina, liječenje peroralnim metotreksatom u dozi od $15 \mathrm{mg} /$ tjedan. Evaluacija motoričke funkcije šake provedena je s pomoću novog ekspertnog elektroničkog mjernog sustava za dobivanje dinamičkih mjerenja sile stiska šake koji je razvijen u Institutu „Ruđer Bošković“ (slika 1.). Umjeravanje uređaja izvedeno je u skladu s usporedbom stiska šake na mehaničkom dinamometrijskom uređaju s istim mehaničkim rukohvatom. Dinamika dinamometrijskih parametara stiska šake za obje je skupine izmjerena na početnome mjerenju i nakon 60, 120 i 180 dana. Svi su bolesnici liječeni temeljnim antireumatskim lijekom koji modificira tijek 
was not changed. The patients had the option of taking NSAIDs and/or acetaminophen as an escape medication, but were required to stop the medication three days before each visit. The main dynamometric parameters of interest obtained were: maximum grip force (highest achieved power of each grip), fatigue (onset of decreasing tendency in maximum grip force in the grip series), grip velocity (ratio of maximum force and time in which the individual grip is achieved), and functional potential (composite measure combining the average grip force, average grip velocity, and fatigue). The evaluation of the hand's functional deficit was performed by registering and extracting previously defined parameters in time and spectral domain over the processed time grip series of the dominant hand. The described pre-processing was conducted over a grip series (15 times) at baseline (moment $t=0)$ and at visits every two months thereafter. In order to minimize fluctuation and amplitude effects, Fourier analysis was used for each cycle. The best 10 from a total of 15 individual cycles were selected for each study participant, and the most irregular cycles, such as those containing more irregular maximums, uneven grip phase, or delay at the beginning and at the end of each grip, were removed. Statistical analysis included the T-test and the ANOVA test for repeated measures.

\section{RESULTS}

The two groups were well matched to age, and no significant difference was found between them (the mean and SD for age in RA patients was $54.7 \pm 8.2$ years, and in healthy controls $54.2 \pm 8.2 ; \mathrm{P}=0.883$ ). The baseline data on the dynamometric parameters of interest in the patients with RA and healthy controls are shown in Table 1. Compared to the control group, the RA patients had a significantly lower maximum grip force, fatigue (earlier occurrence of fatigue), grip velocity, and functional potential.

During the follow-up period, the measured dynamometric parameters in the patients with RA showed significant improvement at each visit as follows: maximum grip force $-\mathrm{P}=0.040$, fatigue $-\mathrm{P}=0.032$, grip velocity $-\mathrm{P}=0.026$, and functional potential $-\mathrm{P}=$ 0.014 (Table 2). Nevertheless, they did not reach the values measured in the healthy controls.

\section{DISCUSSION}

This pilot study presents the results of the application of a new measuring system, involving a unique data processing algorithm and proposed parameters of the hand function. It also considers the implications of this system for rheumatology. Previous studies based on single-grip measurement found that hand grip strength and net forces vary by sex, age, and anthropometric characteristics $(4,5)$. Generally, in conditions affecting the hand the observed dynamometric param-

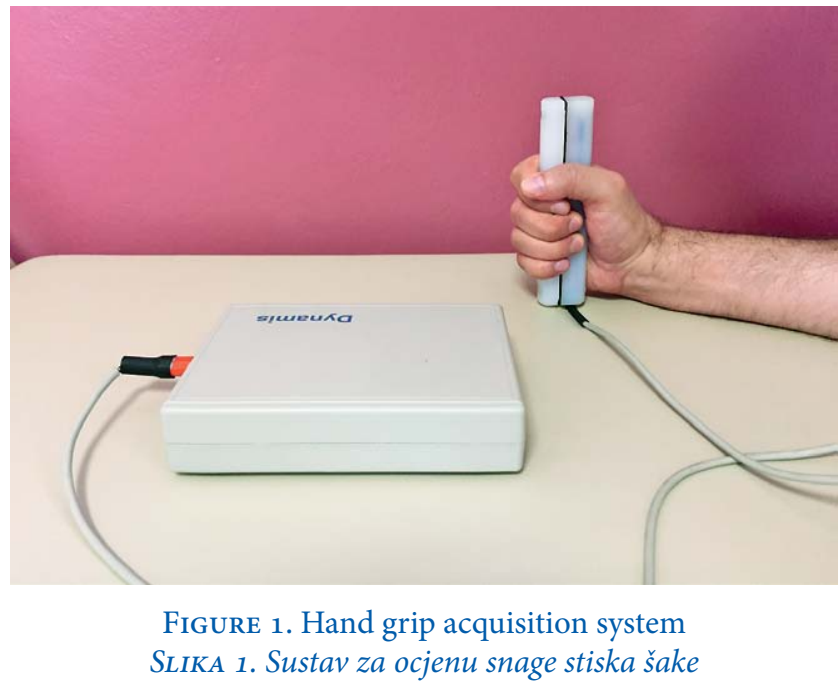

bolesti (DMARD), metotreksatom, a tijekom praćenja njegova doza nije promijenjena. Postojala je i mogućnost uzimanja nesteroidnih antireumatika (NSAR) $\mathrm{i} / \mathrm{ili}$ acetaminofena kao simptomatskog lijeka, ali ne u razdoblju od tri dana prije svake kontrole. Dobiveni glavni dinamometrijski parametri od interesa bili su: maksimalna sila stiska šake (najveća postignuta snaga svakog stiska), zamor šake (trenutak početka tendencije smanjenja maksimalne sile stiska u seriji stisaka), brzina stiska (omjer maksimalne sile i vremena u kojem se postiže pojedinačan stisak) i funkcionalni potencijal (složena mjera koja uključuje prosječnu silu stiska, prosječnu brzinu stiska i prosječan zamor šake). Evaluacija funkcionalnog deficita šake pacijenta provedena je registracijom i izdvajanjem prethodno utvrđenih parametara u vremenskoj i spektralnoj domeni tijekom obrađenih vremenskih serija stiska dominantne šake. Opisana predobrada provedena je na serijama stisaka (15 puta) pri početnomu mjerenju $(\mathrm{t}=0)$ i na kontrolama koje su se provodile svaka dva mjeseca nakon početnog mjerenja. Da bi se utjecaji fluktuacije i amplitude smanjili na minimum, za svaki je ciklus provedena Fourierova analiza. Za svakog je ispitanika odabrano 10 najboljih ciklusa od ukupno 15 pojedinačnih ciklusa, a uklonjeni su najnepravilniji ciklusi poput onih koji su sadržavali više nepravilnijih maksimalnih vrijednosti, neravnomjernu fazu stiska, kašnjenje na početku i na kraju svakog stiska. Statistička analiza uključivala je T-test i analizu varijance (ANOVA) za ponovljena mjerenja.

\section{REZULTATI}

Dvije skupine dobro su se podudarale po dobi, jer između njih nije utvrđena znatna razlika (srednja vrijednost i SD za dob u bolesnika s RA iznosila je 54,7 \pm 8,2 godine, a za kontrolne ispitanike iznosila je $54,2 \pm$ $8,2 ; \mathrm{P}=0,883)$. Podatci $\mathrm{s}$ početnog mjerenja u vezi $\mathrm{s}$ interesnim dinamometrijskim parametrima u bolesnika s RA i kontrolnih ispitanika prikazani su u tablici 1. 
TABLE 1. Differences between patients and control group at baseline (T-test for independent groups)

TABLICA 1. Razlika između bolesnika i kontrolne skupine u odnosu prema početnom stanju (T-test za nezavisne skupine)

\begin{tabular}{|c|c|c|c|c|c|c|c|c|}
\hline & \multicolumn{3}{|c|}{$\begin{array}{l}\text { Control group / Kontrolna skupina } \\
\qquad \mathrm{N}=12\end{array}$} & \multicolumn{3}{|c|}{$\begin{array}{l}\text { Patients / Bolesnici } \\
\quad \mathrm{N}=12\end{array}$} & \multirow[b]{2}{*}{$t$} & \multirow[b]{2}{*}{$p$} \\
\hline & $\begin{array}{c}\text { Mean } \\
\text { / Srednja } \\
\text { vrijednost }\end{array}$ & SD & $\begin{array}{l}\text { Std. Error Mean } \\
\text { / Standardna } \\
\text { pogreška srednje } \\
\text { vrijednosti }\end{array}$ & $\begin{array}{c}\text { Mean } \\
\text { / Srednja } \\
\text { vrijednost }\end{array}$ & SD & $\begin{array}{l}\text { Std. Error Mean } \\
\text { / Standardna } \\
\text { pogreška srednje } \\
\text { vrijednosti }\end{array}$ & & \\
\hline $\begin{array}{l}\text { Max grip force at baseline }(\mathrm{N}) \\
\text { / Maksimalna sila stiska izmjerena } \\
\text { na početnome mjerenju }(\mathrm{N})\end{array}$ & 97,3 & 3,5 & 1,0 & 68,2 & 14,6 & 4,2 & 6,7 & $<0,001$ \\
\hline $\begin{array}{l}\text { Fatigue at baseline / Zamor šake } \\
\text { na početnome mjerenju }\end{array}$ & 24,9 & 0,3 & 0,1 & 22,4 & 1,8 & 0,5 & 4,8 & $<0,001$ \\
\hline $\begin{array}{l}\text { Grip velocity at baseline }(\mathrm{N} / \mathrm{s}) / \text { Brzina } \\
\text { stiska na početnome mjerenju (N/s) }\end{array}$ & 22,2 & 1,3 & 0,4 & 14,8 & 2,0 & 0,6 & 10,7 & $<0,001$ \\
\hline $\begin{array}{l}\text { Functional potential at baseline / Funkcio- } \\
\text { nalni potencijal na početnome mjerenju }\end{array}$ & 1876,3 & 174,1 & 50,2 & 808,2 & 266,0 & 76,8 & 11,6 & $<0,001$ \\
\hline
\end{tabular}

TABLE 2. Changes in dynamometric variables during follow-up - patients with RA ( $n=12)$ (ANOVA) TABLICA 2. Promjene dinamometrijskih varijabla tijekom praćenja-bolesnici s $R A(n=12)$ (analiza varijance, ANOVA)

\begin{tabular}{|c|c|c|c|c|c|}
\hline Patients / Bolesnici N = 12 & $\begin{array}{c}\text { Mean } \\
\text { / Srednja } \\
\text { vrijednost }\end{array}$ & SD & $\begin{array}{l}\text { Wilks' Lambda value } \\
\text { / Wilksova lambda- } \\
\text {-vrijednost }\end{array}$ & $\mathrm{F}$ & $p$ \\
\hline $\begin{array}{l}\text { Max grip force at baseline }(\mathrm{N}) \\
\text { / Maksimalna sila stiska izmjerena na početku }(\mathrm{N})\end{array}$ & 68,2 & 14,6 & \multirow{4}{*}{0,41} & \multirow{4}{*}{4,24} & \multirow{4}{*}{0,040} \\
\hline $\begin{array}{l}\text { Max grip force after } 2 \text { months }(\mathrm{N}) \\
\text { / Maksimalna sila stiska izmjerena nakon } 2 \text { mjeseca }(\mathrm{N})\end{array}$ & 67,4 & 12,4 & & & \\
\hline $\begin{array}{l}\text { Max grip force after } 4 \text { months }(\mathrm{N}) \\
\text { / Maksimalna sila stiska izmjerena nakon } 4 \text { mjeseca }(\mathrm{N})\end{array}$ & 71,1 & 14,1 & & & \\
\hline $\begin{array}{l}\text { Max grip force after } 6 \text { months }(\mathrm{N}) \\
\text { / Maksimalna sila stiska izmjerena nakon } 6 \text { mjeseci }(\mathrm{N})\end{array}$ & 71,8 & 13,6 & & & \\
\hline Fatigue at baseline / Zamor šake izmjeren na početku & 22,4 & 1,8 & \multirow{4}{*}{0,39} & \multirow{4}{*}{4,63} & \multirow{4}{*}{0,032} \\
\hline Fatigue after 2 months / Zamor šake izmjeren nakon 2 mjeseca & 23,2 & 1,5 & & & \\
\hline Fatigue after 4 months / Zamor šake izmjeren nakon 4 mjeseca & 23,7 & 1,4 & & & \\
\hline Fatigue after 6 months / Zamor šake izmjeren nakon 6 mjeseci & 23,8 & 1,6 & & & \\
\hline Grip velocity at baseline (N/s) / Brzina stiska izmjerena na početku (N/s) & 14,8 & 2,0 & \multirow{4}{*}{0,38} & \multirow{4}{*}{4,97} & \multirow{4}{*}{0,026} \\
\hline Grip velocity after 2 months (N/s) / Brzina stiska izmjerena nakon 2 mjeseca (N/s) & 14,8 & 2,3 & & & \\
\hline Grip velocity after 4 months (N/s) / Brzina stiska izmjerena nakon 4 mjeseca (N/s) & 15,9 & 3,1 & & & \\
\hline $\begin{array}{l}\text { Grip velocity after } 6 \text { months }(\mathrm{N} / \mathrm{s}) \\
\text { / Brzina stiska izmjerena nakon } 6 \text { mjeseci }(\mathrm{N} / \mathrm{s})\end{array}$ & 16,7 & 3,1 & & & \\
\hline Functional potential at baseline / Funkcionalni potencijal izmjeren na početku & 808,2 & 266,0 & \multirow{4}{*}{0,32} & \multirow{4}{*}{6,25} & \multirow{4}{*}{0,014} \\
\hline $\begin{array}{l}\text { Functional potential after } 2 \text { months } \\
\text { / Funkcionalni potencijal izmjeren nakon } 2 \text { mjeseca }\end{array}$ & 831,9 & 251,6 & & & \\
\hline $\begin{array}{l}\text { Functional potential after } 4 \text { months } \\
\text { / Funkcionalni potencijal izmjeren nakon } 4 \text { mjeseca }\end{array}$ & 964,0 & 332,1 & & & \\
\hline $\begin{array}{l}\text { Functional potential after } 6 \text { months } \\
\text { / Funkcionalni potencijal izmjeren nakon } 6 \text { mjeseci }\end{array}$ & 1027,4 & 340,1 & & & \\
\hline
\end{tabular}

eters increased, as strength, velocity, and grip stability grew (2). Compared to the baseline values, the increase in these parameters during the treatment represented a clear indicator of improvement of the hand function. The meticolous evaluation of the hand function using this new dynamometric system offers the possibility to plan appropriate exercises in order to obtain the best possible result. During a period of 6 months, the method showed good resolution at all stages.
U usporedbi s kontrolnom skupinom, bolesnici s RA imali su znatno slabiju maksimalnu silu stiska šake, manju vrijednost zamora šake (zamor se dogodio puno prije nego u kontrolnih ispitanika), manju brzinu stiska i manji funkcionalni potencijal.

Tijekom praćenja pri svakoj kontroli utvrđene su promjene $\mathrm{u}$ izmjerenim dinamometrijskim parametrima $\mathrm{u}$ bolesnika s RA: maksimalna sila stiska šake $(P=0,040)$, zamor šake $(P=0,032)$, brzina stiska $(P=0,026)$ i funk- 
It is important to note that within the framework of this study, data on grip force and velocity are primarily significant in relative relations. Hence, they should be considered with caution, whereas for accurate absolute values of the abovementioned parameters it would be necessary to perform a study on a larger number of subjects and with a stringently defined protocol of device calibration (6). The development of this expert diagnostic system could allow an objective hand functional status evaluation. For further research, along with net functional force and velocity in hand grip evaluation, and with adequate probe modification, it would also be possible to analyze the grip force of a specific group of digits - mostly that of the thumb plus one finger ("pinch testing").

\section{CONCLUSION}

In this pilot evaluation study we present a new electronic dynamometric system for obtaining hand function parameters based on dynamic measurements. By comparing the results of healthy subjects and patients, and by recording the changes occurring during the treatment, we can conclude that this system measures the hand condition objectively. It can be safely used to monitor the status of individual patients during treatment and rehabilitation as a relevant indicator of recovery. Further studies with a larger number of patients are needed to confirm its value.

Conflict of interest statement: Authors declare no conflict of interest.

\section{REFERENCES / LITERATURA}

1. Sheehy C, Gaffney K, Mukhtyar C. Standardized grip strength as an outcome measure in early rheumatoid arthritis. Scand J Rheumatol. 2013;42(4):289-93.

2. Dias JJ, Singh HP, Taub N, Thompson J. Grip strength characteristics using force-time curves in rheumatoid hands. J Hand Surg Eur Vol. 2013;38(2):170-7.

3. Björk M, Thyberg I, Haglund L, Skogh T. Hand function in women and men with early rheumatoid arthritis. A prospective study over three years (the Swedish TIRA project). Scand J Rheumatol. 2006;35(1):15-9.

4. Beenakker KGM, Ling CH, Meskers CGM i sur. Patterns of muscle strength loss with age in the general population and patients with a chronic inflammatory state. Ageing Res Rev. 2010;9(4):431-6. doi: 10.1016/j.arr.2010.05.005.

5. Chandrasekaran B, Ghosh A, Prasad C i sur. Age and anthropometric traits predict handgrip strength in healthy normals. J Hand Microsurg. 2010;2(2):58-61. doi: 10.1007/s12593-0100015-6.

6. Shiratori AP, da Rosa Iop R, Gomes Borges Júnior N i sur. Evaluation protocols of hand grip strength in individuals with rheumatoid arthritis: a systematic review. Rev Bras Reumatol. 2014;54(2):140-7. cionalni potencijal $(\mathrm{P}=0,014)$ (tablica 2.). No, bez obzira na prije navedeno, bolesnici s RA nisu uspjeli postići jednake rezultate kao kontrolni ispitanici.

\section{RASPRAVA}

U ovoj smo pilot studiji, s pomoću jedinstvenog algoritma obrade podataka i predloženih parametara za mjerenje funkcije šake, opisali primjenu novog sustava mjerenja i njegovo značenje u reumatologiji. $\mathrm{U}$ prije provedenim studijama, koje su se temeljile na mjerenju pojedinačnog stiska šake, utvrđeno je da se snaga stiska šake i neto sila razlikuju ovisno o spolu, dobi i antropometrijskim značajkama $(4,5)$. Općenito se, u uvjetima koji utječu na funkciju šake, promatrani dinamometrijski parametri povećavaju s povećanjem snage, brzine i stabilnosti stiska šake (2). U usporedbi s početnim vrijednostima mjerenja, povećanje tih parametara tijekom liječenja jasno upućuje na poboljšanje funkcije šake. Precizna evaluacija funkcije šake koja se provodi s pomoću ovoga novog dinamometrijskog sustava pruža mogućnost planiranja odgovarajućih vježbi s ciljem dobivanja najboljih mogućih rezultata. Ovom metodom dobiveni su dobri rezultati u svim fazama tijekom razdoblja od 6 mjeseci.

Valja napomenuti da u ovoj studiji podatci o sili i brzini stiska imaju primarnu važnost u okviru relativnih odnosa parametara. Stoga ih treba uzimati s oprezom, dok bi za točne apsolutne vrijednosti gore navedenih parametara trebalo provesti istraživanje na većem broju ispitanika i sa strogo utvrđenim protokolom umjeravanja uređaja (6). Razvoj ovog ekspertnog dijagnostičkog sustava omogućio bi provođenje objektivne evaluacije funkcionalnog statusa šake. $U$ daljnjim se istraživanjima, uz odgovarajuću modifikaciju sonde, zajedno s neto funkcionalnom silom i brzinom u evaluaciji stiska šake može analizirati i sila stiska šake određene skupine prstiju - uglavnom sila palca i jednoga dodatnog prsta (ispitivanje snage stiska prstima) (engl. pinch testing).

\section{ZAKLJUČAK}

U ovoj smo pilot evaluacijskoj studiji predstavili novi elektronički dinamometrijski sustav za dobivanje parametara funkcije šake koji se temelje na dinamičkim mjerenjima. Usporedbom rezultata u zdravih kontrolnih ispitanika i u bolesnika s RA te bilježenjem promjena tijekom liječenja možemo zaključiti da ovaj sustav objektivno mjeri stanje šake. Sustav je siguran za uporabu u praćenju stanja pojedinih bolesnika tijekom liječenja i rehabilitacije, kao bitan pokazatelj njihova oporavka. Potrebno je provesti daljnje studije na većem broju bolesnika da bi se potvrdile vrijednost i učinkovitost ovog uređaja.

Izjava o sukobu interesa: Autori izjavljuju da nisu u sukobu interesa. 